\title{
TAZ is required for chondrogenesis and skeletal development
}

\author{
Yang Li', Shuting Yang ${ }^{1}$, Ling Qin² and Shuying Yang $\mathbb{B D}^{1,3,4}$
}

\begin{abstract}
Chondrogenesis is a major contributor to skeletal development and maintenance, as well as bone repair. Transcriptional coactivator with PDZ-binding motif (TAZ) is a key regulator of osteogenesis and adipogenesis, but how TAZ regulates chondrogenesis and skeletal development remains undefined. Here, we found that TAZ expression is gradually increased during chondrogenic differentiation. Deletion of TAZ in chondrocyte lineage impaired articular and growth plate, as well as the bone development in TAZ-deficient mice. Consistently, loss of TAZ impaired fracture healing. Mechanistically, we found that ectopic expression of TAZ markedly promoted chondroprogenitor proliferation, while deletion of TAZ impaired chondrocyte proliferation and differentiation. TAZ associated with Sox 5 to regulate the expression and stability of Sox 5 and downstream chondrocyte marker genes' expression. In addition, overexpression of TAZ enhanced Col10a1 expression and promoted chondrocyte maturation, which was blocked by deletion of TAZ. Overall, our findings demonstrated that TAZ is required for skeletal development and joint maintenance that provided new insights into therapeutic strategies for fracture healing, heterotopic ossification, osteoarthritis, and other bone diseases.
\end{abstract}

\section{Introduction}

Chondrogenesis is a major process during endochondral ossification that leads to the skeletal formation and growth, as well as repair from mesenchymal stem cells (MSCs) in adults ${ }^{1-3}$. It has been reported that endochondral bone formation accounts for over $80 \%$ of the skeleton volume ${ }^{4}$. Endochondral ossification is initiated with the formation of cartilage templates of the future bone by mesenchymal progenitor cells. Those cells condensate and differentiate into chondrocytes, and then develop into the growth plate cartilage wherein chondrocytes could drive longitudinal bone growth by their proliferation, differentiation, and mineralization ${ }^{5,6}$. Dysfunction of chondrogenesis and skeleton development often causes numerous bone diseases, such as skeleton

\footnotetext{
Correspondence: Shuying Yang (shuyingy@upenn.edu)

'Department of Basic \& Translational Sciences, School of Dental Medicine, University of Pennsylvania, Philadelphia, PA 19104, USA

${ }^{2}$ Department of Orthopedic Surgery, Perelman School of Medicine, University of Pennsylvania, Philadelphia, PA 19104, USA
}

Full list of author information is available at the end of the article abnormalities, heterotopic ossification, defective fracture healing, and osteoarthritis $(\mathrm{OA})^{7}$, which currently lack of effective drugs ${ }^{8,9}$. Understanding the molecular and signaling basis underlying the influence of target genes on chondrocytes and cartilage will provide the foundation for the development of therapeutic paradigms to ameliorate the impact of these bone diseases.

Chondrogenic differentiation of MSCs has been well established, which makes these cells a highly promising candidate for cartilage tissue repair ${ }^{10,11}$. Numerous studies reported that the chondrogenic differentiation required the involvement of multiple factors both in vivo and in vitro of chondrogenesis and skeletal development $^{12}$. For instance, aggrecan and collagen II (Col-II), two cartilage extracellular matrix proteins, are commonly accepted as proliferative chondrogenic-specific markers. In hypertrophic chondrocytes, Mmp13 and Col10a1 are considered as key mediators of chondrogenic differentiation. Previous studies have also reported that SRY-related high-mobility group box (Sox) genes (Sox5/6/9), Runtrelated transcription factor 2 (Runx2), and Indian 
hedgehog (Ihh) are well-known regulators of chondrogenesis ${ }^{13-16}$. Although previous studies have investigated chondrogenic differentiation, the precise molecular mechanisms controling the chondrogenic differentiation are still largely undefined.

Recently, the Hippo pathway has been established as a critical regulator of organ size, tissue repair, and regeneration; cell proliferation, apoptosis, autophagy, and tumorigenesis ${ }^{17-19}$. It also plays critical roles in regulating osteogenesis and bone development, as well as the progression of various diseases, including $\mathrm{OA}$ and bone cancer $^{20,21}$. Transcriptional coactivator with a PDZbinding domain (TAZ), a core downstream effector of the Hippo pathway, is a key regulator maintaining the balance between osteogenesis and adipogenesis ${ }^{22,23}$. For instance, nuclear translocation of TAZ directly interacts with Runx2 to stimulate osteogenesis, and represses PPAR $\gamma$ transcriptional activity and adipogenesis ${ }^{24,25}$. Moreover, transgenic overexpression of TAZ in osteoblasts results in an increase of osteoblast-mediated bone formation and a decrease of bone marrow adipogenesis $^{22,26}$. In contrast, depletion of TAZ in zebrafish or in osteoblast precursors in mice impairs the bone development $^{22,27,28}$. In addition, global TAZ knockout mice exhibit small stature and ossification defects ${ }^{29}$. Besides, Yes-associated protein (YAP), a paralog of TAZ and another effector of the Hippo pathway, has been reported to regulate multiple steps of chondrocyte differentiation during skeletal development and bone repair; however, different from TAZ, YAP cannot bind with PPARy to direct adipogenesis directly ${ }^{12,24,30,31}$. Thus, it is important to determine whether and how TAZ regulates chondrogenesis, cartilage development, and homeostasis, which is currently unknown.

In this study, we determined the expression pattern of TAZ during chondrocyte differentiation, and characterized the contribution of TAZ to chondrocyte proliferation, differentiation and maturation, and skeleton development. We generated Col2-Cre; $\mathrm{TAZ}^{\mathrm{f} / \mathrm{f}}$ by crossing TAZ floxed mice and Col2a1-Cre transgenic mice, and determined the function and mechanism of TAZ in the regulation of chondrogenesis and cartilage and bone development, using loss- and gain-of-function approaches. We found that TAZ is required for chondrogenesis and skeletal development and repair.

\section{Results}

TAZ expression is gradually increased during chondrogenic differentiation

Heatmap of publicly available expression data from early murine limb development (from E9.5 to E13.5; GSE30138 ${ }^{32}$ revealed a TAZ conserved signature as one of the top significantly enriched developmental gene sets (Fig. 1a). Consistently, we found the expression levels of negative regulators (MST1, LATS1, and MOB1) of TAZ activity decreased, while TAZ target genes (CTGF and CY61) increased during chondrogenic differentiation (Fig. 1a). In addition, we found that YAP expression decreased during chondrogenic differentiation. To explore the function of TAZ in the cartilage development, we further identified the endogenous expression pattern of TAZ in the tibia growth plate of wild-type mice (WT) at E16.5
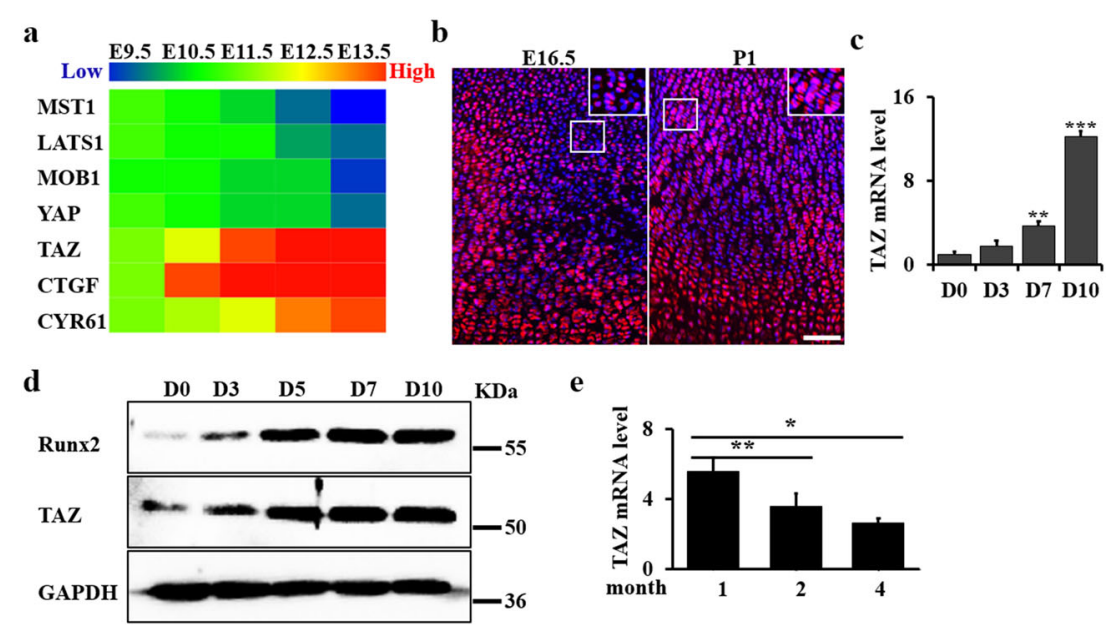

Fig. 1 TAZ expression is gradually increased during chondrogenic differentiation. a Heatmap analysis of TAZ-associated genes in early murine limb (from E9.5 to E13.5; GSE3013832). b Representative immunofluorescence-stained images of tibiae from WT mice at the ages of E16.5 and P1, labeled for TAZ and stained for DAPI. Scale bars, $75 \mu \mathrm{m}$. c qRT-PCR analysis of the chondrocyte differentiation markers of micromass culture as indicated time. $\mathbf{d}$ Whole-cell lysates from micromass culture were immunoblotted with antibodies against TAZ, Runx2, and GAPDH. e qRT-PCR analysis of TAZ expression using the articular cartilage from the WT mice at 1, 2, and 4 months. The experiment was independently repeated for three times. Error bars were the means \pm SEM of triplicates from a representative experiment. ${ }^{*} P<0.05,{ }^{* *} P<0.01,{ }^{* * *} P<0.001$. 
and postnatal day 1 (P1). We found that TAZ expression gradually increased from E16.5 to P1 (Fig. 1b). To investigate the expression and function of TAZ in chondrocyte differentiation, we performed the micromass culture as an in vitro model for cell differentiation, using primary chondrocytes isolated from the limb buds of WT embryos at E15.5 (Supplementary Fig. S1a). We confirmed that the chondrocyte differentiation markers, including $M M P 13$, Runx2, Ihh, Col2a1, Col10a1, and Aggrecan, increased and peaked at approximately day 7 to day 10 after chondrogenic induction (Supplementary Fig. S1b). Interestingly, we found that the expression levels of TAZ in chondrocytes were concomitant with chondrocyte differentiation (Fig. 1c). Western blot analysis further confirmed that the expression of TAZ was gradually increased during the chondrocyte differentiation (Fig. 1d). However, we found the TAZ expression gradually decreased from 1 month to 4 months in the articular cartilage (Fig. 1e).

\section{Deletion of TAZ in chondrocytes inhibits growth plate and articular cartilage development}

To further investigate the potential contribution of TAZ to chondrogenesis, we generated a mutant line in which TAZ was deleted in chondrocyte lineage through crossing $\mathrm{TAZ}^{\mathrm{f} / \mathrm{f}}$ mice with a transgenic Cre line driven by a Col2a1 promoter (henceforth referred to as Col2-Cre;TAZ ${ }^{\mathrm{f} / \mathrm{f}}$ mice). qRT-PCR and western blot results showed an effective deletion of TAZ in the cartilage of Col2-Cre; $\mathrm{TAZ}^{\mathrm{f} / \mathrm{f}}$ mice compared to controls (TAZ ${ }^{\mathrm{f} / \mathrm{f}}$; Supplementary Fig. S2a, b); however, the cortical bone had no pronounced change (Supplementary Fig. S2c, d). Col2-Cre; $\mathrm{TAZ}^{\mathrm{f} / \mathrm{f}}$ newborn pups displayed the growth retardation and shorter limbs (Fig. 2a, b). Alcian blue/Alizarin Red staining confirmed that the Col2-Cre;TAZ ${ }^{\mathrm{f} / \mathrm{f}}$ mice displayed underdeveloped calvarium, hypoplastic ribs, sternum, and shorter limb bones (Fig. 2a-c). Moreover, histologic examination of tibiae revealed that the proliferation zone, prehypertrophic zone, and hypertrophic zone were all shorter in the growth plate of Col2-Cre; $\mathrm{TAZ}^{\mathrm{f} / \mathrm{f}}$ mice, compared to those in controls (Fig. $2 \mathrm{~d}-\mathrm{f}$ ). In addition, growth plates in the long bones of Col2-Cre; $\mathrm{TAZ}^{\mathrm{f} / \mathrm{f}}$ mice were shorter than those in the control mice (Fig. $2 \mathrm{~g}-\mathrm{i}$ ). To evaluate the effect of TAZ on articular cartilage formation and maintenance, we used Safranin O staining to detect the articular cartilage integrity. The results showed that the width of articular cartilages in 1month-old TAZ-deficient mice was thicker (blue arrows) than that in the controls (Fig. 2j, upper panel; Supplementary Fig. S3a). Interestingly, at 4-month-old, articular cartilage showed an apparent damage in TAZ-deficient mice (yellow arrows) compared to the control (Fig. 2j, lower panel). To further test how TAZ affects the expression of genes in matrix synthesis (Col2a1 and Acan) and hypertrophy (Mmp13 and Runx2) in cartilage, we performed RT-PCR and found that loss of TAZ decreased the expression levels of the chondrocyte markers including Col2a1, Col9a1, Col10a1, Acan, and Mmp13 (Fig. 2k). Consistent with the lower expression of TAZ at 4 months compared to 1 month, the inhibitory effect of TAZ on the Col10a1 expression was reduced at 4 months compared to that at 1 month (Supplementary Fig. S3b), suggesting that TAZ may play a complicated role during the skeletal development. Overall, these results demonstrated that TAZ is required for the development of growth plate and cartilage.

\section{Deletion of TAZ in chondrocyte impairs bone development}

To determine whether the deletion of TAZ in chondrocytes affects bone development, we examined 1month-old Col2-Cre; $\mathrm{TAZ}^{\mathrm{f} / \mathrm{f}}$ mice and controls by microcomputed tomography $(\mathrm{CT})$. We found the bone mass was decreased in the TAZ-deficient mice. BV/TV, trabecular number, and trabecular thickness decreased 1.77-, $1.65-$, and 1.59 -folds, respectively, and trabecular separation increased 1.74-folds compared to those in the control (Fig. 3a-e). Von Kossa staining result showed an obvious reduction in the bone mineralization in long bone of E18.5 Col2-Cre; $\mathrm{TAZ}^{\mathrm{f} / \mathrm{f}}$ mice compared to those in the control mice (Fig. 3f). Analysis of tibia cancellous bone by histomorphometry showed that the loss of TAZ decreased the dynamic indices of bone formation mineral apposition rate (MAR) and bone formation rate (BFR) in Col2-Cre; TAZ ${ }^{\mathrm{f} / \mathrm{f}}$ mice (Fig. 3g-i). Taken together, these data demonstrated that TAZ is required for the bone development.

\section{TAZ is required for chondroprogenitor cell proliferation}

To study the mechanistic actions of TAZ during cartilage and bone development, we first explored the effect of TAZ on chondrocyte proliferation by deletion of TAZ in chondrocytes isolated from Col2-Cre;TAZ ${ }^{\mathrm{f} / \mathrm{f}}$ mice. The proliferation rate of chondrocytes was greatly decreased when TAZ was deleted for day (D) 1, 2, and 3 (Fig. 4a). To further confirm the relationship between TAZ expression and chondroprogenitor proliferation, TAZ was silenced with shTAZ lentiviruses (Supplementary Fig. S4a) or overexpressed with pCDNA3.1-TAZ vector in WT primary chondrocytes. As shown in Fig. 4b, c, silence of TAZ inhibited, while ectopic expression of TAZ markedly promoted chondroprogenitor proliferation. Concomitantly, the number of colonies in TAZ-deficient cells also remarkably decreased compared to the control cells (Fig. 4d). In vivo, a 5-bromo-2'-deoxyuridine (BrdU) labeling assay showed much fewer proliferating cells in the growth plate of 10-day-old Col2-Cre; $\mathrm{TAZ}^{\mathrm{f} / \mathrm{f}}$ mouse tibia compared to the control (Fig. 4e). To further identify mechanisms of the TAZ regulation in chondrocytes, we examined the cartilage of 1-month-old mice because severe lesions had not yet developed at this time. 


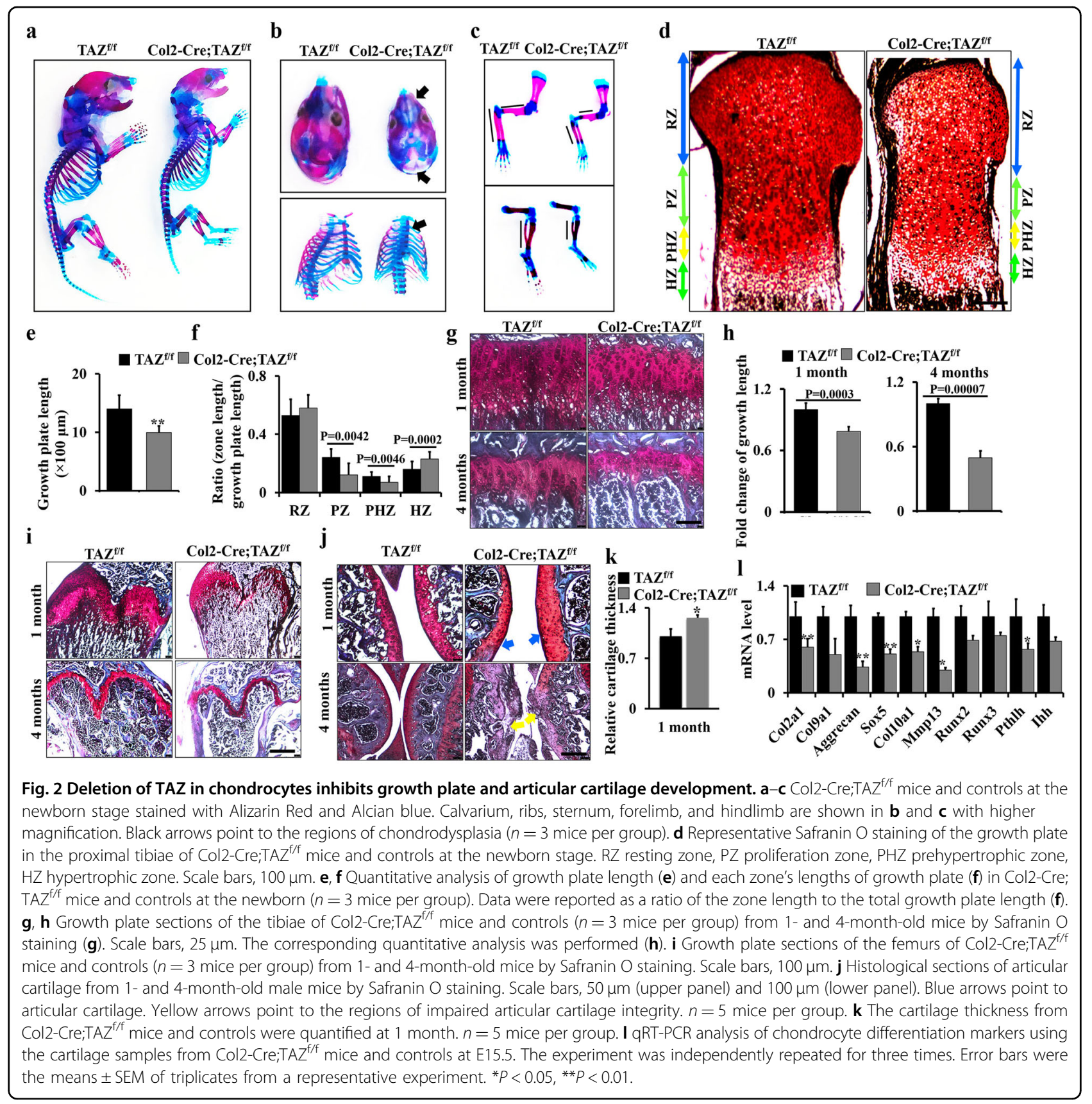

Terminal deoxynucleotidyl transferase-mediated deoxyuridine triphosphate nick-end labeling (TUNEL) staining revealed a marked decrease in the number of apoptotic chondrocytes in the articular cartilage of Col2-Cre; TAZ ${ }^{\mathrm{f} / \mathrm{f}}$ mice (Fig. 4f). All these data indicated that TAZ is required for chondroprogenitor cell proliferation.

\section{TAZ partners with TEAD1 to regulate Sox 5 expression in chondrocytes}

It is well known that TAZ controls stemness by regulating the nucleocytoplasmic shuttling of some transcriptional factors, such as Smads and Snail/Slug ${ }^{33,34}$. Sox 5 has been reported to play a critical role in chondrocyte differentiation. It interacts with YAP, a paralog of TAZ, to drive the malignant potential of non-small cell lung cancer cells by promoting proliferation, migration and invasion, and sustains their self-renewal in tumors ${ }^{35-37}$. To test whether TAZ affects chondrocytes through regulating Sox 5 transcriptional factor, we performed western blot and immunofluorescence staining. As expected, we found that TAZ associate with Sox 5 in the nucleus (Fig. 5a, b). Significantly decreased expression of TAZ target genes, including Cyr61, 
a

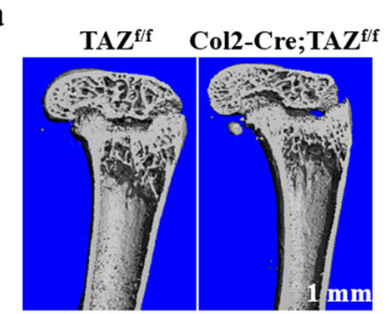

d

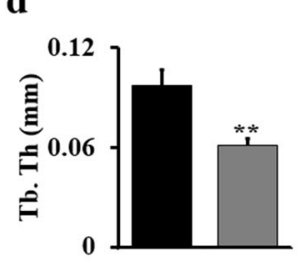

g

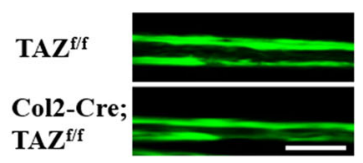

b

e

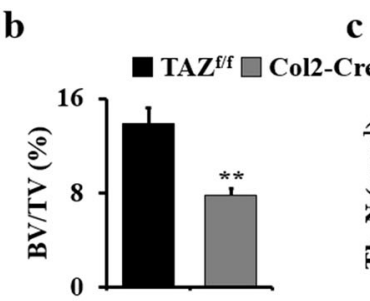

c

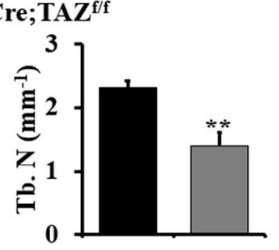

f $\quad \mathrm{TAZ}^{\mathrm{f} / \mathrm{f}} \quad$ Col2-Cre; $T A Z^{\mathrm{f} / \mathrm{f}}$

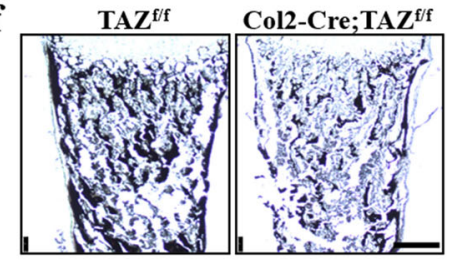

h

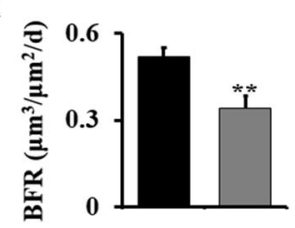

i

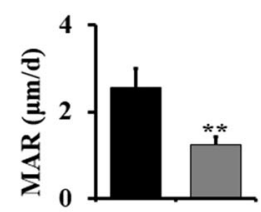

Fig. 3 Deletion of TAZ in chondrocytes impairs bone development. a Representative micro-CT reconstructions of the femurs of Col2-Cre;TAZ $Z^{f / f}$ mice and controls at 1 month. Scale bars, $1 \mathrm{~mm}$. b-e Histomorphometric analysis of bone parameters in the femur of Col2-Cre; TAZ $Z^{\mathrm{f} / \mathrm{f}}$ and control mice ( $n=5$ mice per group). Bone volume fraction (BV/TV) (b); trabecular thickness (Tb.Th) (c); trabecular number (Tb.N) (d); trabecular spacing (Tb. Sp) (e). f Representative von Kossa staining of terminally differentiated hypertrophic chondrocytes in the tibiae of mice at E18.5 ( $n=3$ mice per group). Scale bars, $75 \mu \mathrm{m}$. g-i Calcein double labeling in tibia of 2-month-old Col2-Cre; TAZ ${ }^{\mathrm{f} / \mathrm{f}}$ mice and controls. The mice were injected with calcein twice with an interval of 7 days. The mice were sacrificed 1 day after the second injection. The tibia bones were embedded, sectioned, and the images were taken using a microscope. $\mathbf{h}$ Bone formation rate (BFR); $\mathbf{i}$ mineral apposition rate (MAR). $n=5$ mice per group. The experiment was repeated three times independently. Error bars were the means \pm SEM of triplicates from a representative experiment. ${ }^{* *} P<0.01$.

$C T G F$, and BNDF was observed in Sox5-silenced chondrocytes (Supplementary Fig. S4a-c). Forced expression of Sox 5 rescued the proliferation of TAZ-silenced cells, indicating that TAZ affects cell proliferation by regulating Sox 5 (Fig. 5c). To further understand the regulation of TAZ in Sox5, we next performed western blot and immunofluorescence staining assays to identify the expression of Sox 5 in Col2-Cre;TAZ ${ }^{\mathrm{f} / \mathrm{f}}$ mice and controls. We found the deletion of TAZ resulted in a significant reduction in Sox 5 expression level in vivo (Fig. $5 \mathrm{~d}$, e). To further determine whether Sox 5 expression is required for TAZ in chondrocytes, we tested Sox 5 expression in the in vitro primary chondrocyte culture system. Consistent with the in vivo findings, overexpression or silence of TAZ significantly upregulated or downregulated the expression of Sox 5 (Fig. $5 \mathrm{f}$ and Supplementary Fig. S4d, e). Interestingly, when chondrocytes were treated with the protein synthesis inhibitor cycloheximide $(\mathrm{CHX}, 50 \mu \mathrm{g} / \mathrm{mL})$, we found that overexpression of TAZ increased the expression level of Sox 5 compared to the control, suggesting that TAZ can promote Sox 5 stability (Fig. 5g and Supplementary Fig. S5a). To get further insight into the regulation of TAZ in Sox 5 signaling, we analyzed the binding motif of
TAZ-TEAD1 complex in the Sox5 promoter using Vector NTI software. As expected, we found a binding site of TAZ-TEAD1 complex in the promoter region of the Sox 5 gene (Fig. 5h). We therefore carried out chromatin immunoprecipitation (ChIP) assays using primary chondrocytes isolated from Col2-Cre; $\mathrm{TAZ}^{\mathrm{f} / \mathrm{f}}$ mice and controls. The specific DNA-binding regions of TAZ/TEAD1-Sox 5 was amplified by qRT-PCR after immunoprecipitation with TAZ antibodies. As shown in Fig. 5i, the transcriptional activity of the Sox5 promoter significantly decreased after deletion of TAZ, whereas the transcriptional activity of the Sox5 promoter was significantly blocked after silence of TEAD 1 by TEAD1 siRNA in chondrocytes (Fig. $5 \mathrm{j}$ and Supplementary Fig. S5b). In addition, TAZ overexpression could also increase the Sox5 transcriptional activity (Fig. 5k). Overall, these data demonstrated that TAZ partners with TEAD1 to regulate the Sox 5 expression in chondrocytes.

\section{Deletion of TAZ blocks chondrocyte maturation via inhibiting Col10a1 expression}

To test whether TAZ is also involved in chondrocyte maturation, we cultured primary chondrocytes from Col2-Cre;TAZ ${ }^{\mathrm{f} / \mathrm{f}}$ mice and controls in chondrogenic 


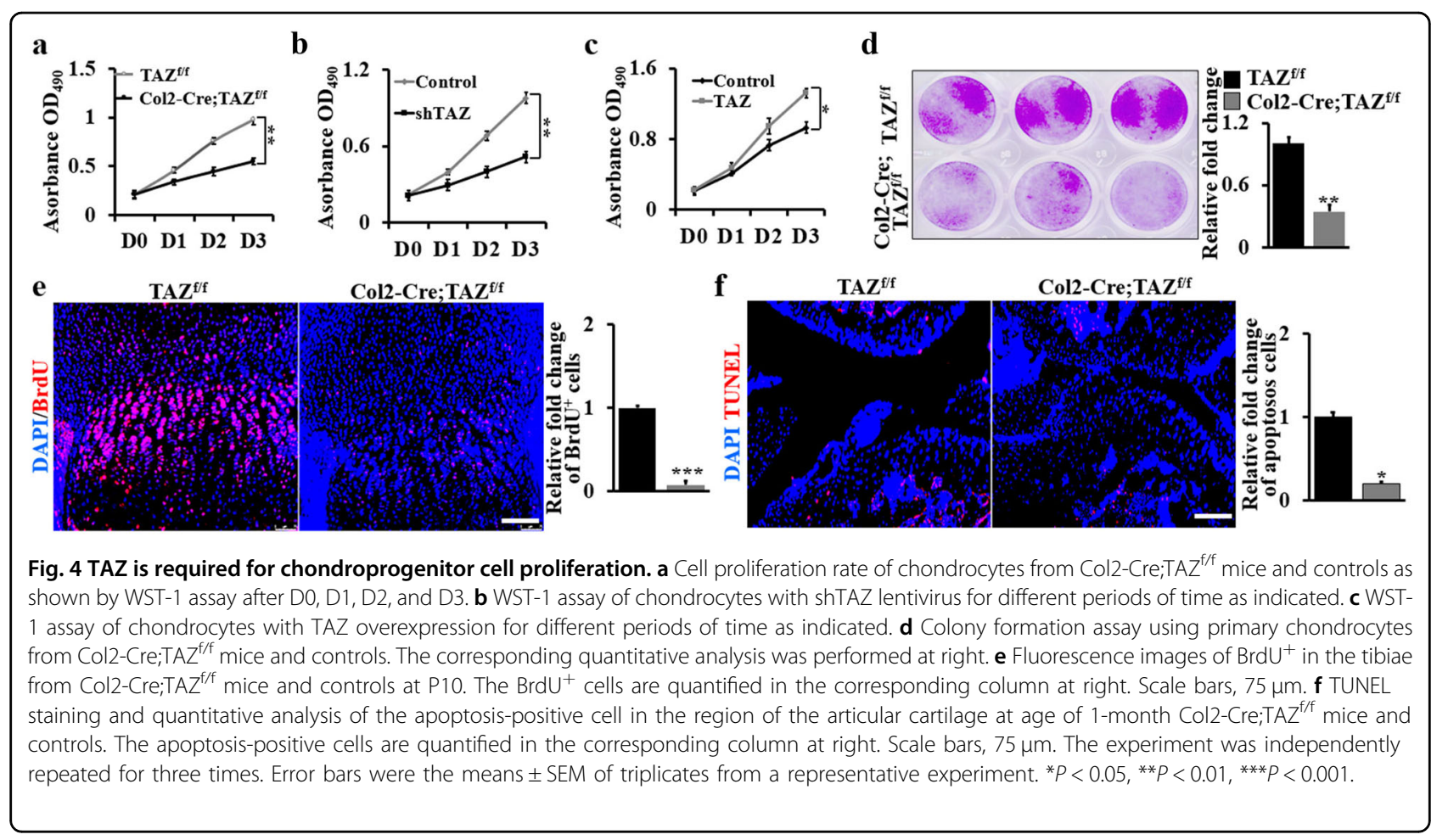

differentiation medium for 7 days. Interestingly, micromass culture showed that the loss of TAZ decreased cartilage nodule formation (Fig. 6a). Next, we identified the expression level of the hypertrophic chondrocyte marker Col10a1 after culturing chondrocytes in chondrogenic medium for 7 days. We found that the loss of TAZ significantly inhibited Col10a1 expression by qRTPCR (Fig. 6b). Moreover, silence of TAZ in chondrocytes infected with shTAZ lentivirus showed similar results (Fig. 6c). In contrast, the overexpression of TAZ increased Col10a1 expression (Fig. 6d). Previous studies showed that Runx2 is a major regulator of chondrocyte hypertrophy that promotes the maturation of chondrocytes by regulating the expression of its target gene Col10a $1^{12,38}$. Yoshinori Matsumoto et al. reported that TAZ directly interacts with Runx2 to regulate bone development ${ }^{23}$. Consistent with those findings, immunofluorescence staining analysis further confirmed that the Runx2 expression level was much lower in chondrocytes from Col2-Cre; $\mathrm{TAZ}^{\mathrm{f} / \mathrm{f}}$ compared to those from control mice (Fig. 6e). Western blot assays in primary chondrocyte culture further confirmed this result in vitro (Fig. $6 \mathrm{f}$ and Supplementary Fig. S5c). To further test whether TAZ directly affects chondrocyte maturation, we next determined whether overexpression of Runx 2 affects Col10a1 expression in TAZ-dependent manner. Interestingly, we found that the overexpression of Runx2 increased Col10a1 expression, which was blocked by silencing TAZ, but significantly enhanced by TAZ overexpression
(Fig. 6g, h), suggesting the Col10a expression regulated by Runx2 is required for TAZ signaling. It has been reported that Runx2 has three DNA-binding sites in the promoter of $\mathrm{CollOa}^{12,38}$. Therefore, to further understand the relationship between TAZ, Runx2, and Col10a, we carried out a ChIP assay using Col10a1 promoter region where the three TAZ/Runx2 complex binding sites were found (Fig. 6i). Our data showed that the transcriptional activity of the Col10a1 promoter increased with TAZ overexpression (Fig. 6j). Taken together, our results suggested that TAZ promotes chondrogenic maturation by regulating Col10a1 expression.

\section{Deletion of TAZ in chondrocytes impaired the fracture healing}

Chondrocyte differentiation and maturation play important roles in bone repair and fracture healing. To gain further insights into TAZ function in the postnatal endochondral ossification, we created closed mid-shaft femur fracture model in Col2-Cre; $\mathrm{TAZ}^{\mathrm{f} / \mathrm{f}}$ and control mice that generates a robust endochondral healing response. After 2-week bone fracture healing, the X-ray result showed obvious callus formation at the fracture site of control $\mathrm{TAZ}^{\mathrm{f} / \mathrm{f}}$ mice, with a significant amount of cartilage formation, whereas cartilaginous callus formation in the Col2-Cre;TAZ ${ }^{\mathrm{f} / \mathrm{f}}$ mice was significantly impaired (Fig. 7a). Consistently, micro-CT results also showed less bone formation in Col2-Cre; $\mathrm{TAZ}^{\mathrm{f} / \mathrm{f}}$ mice compared to that in controls (Fig. 7b); and the surface and volume 


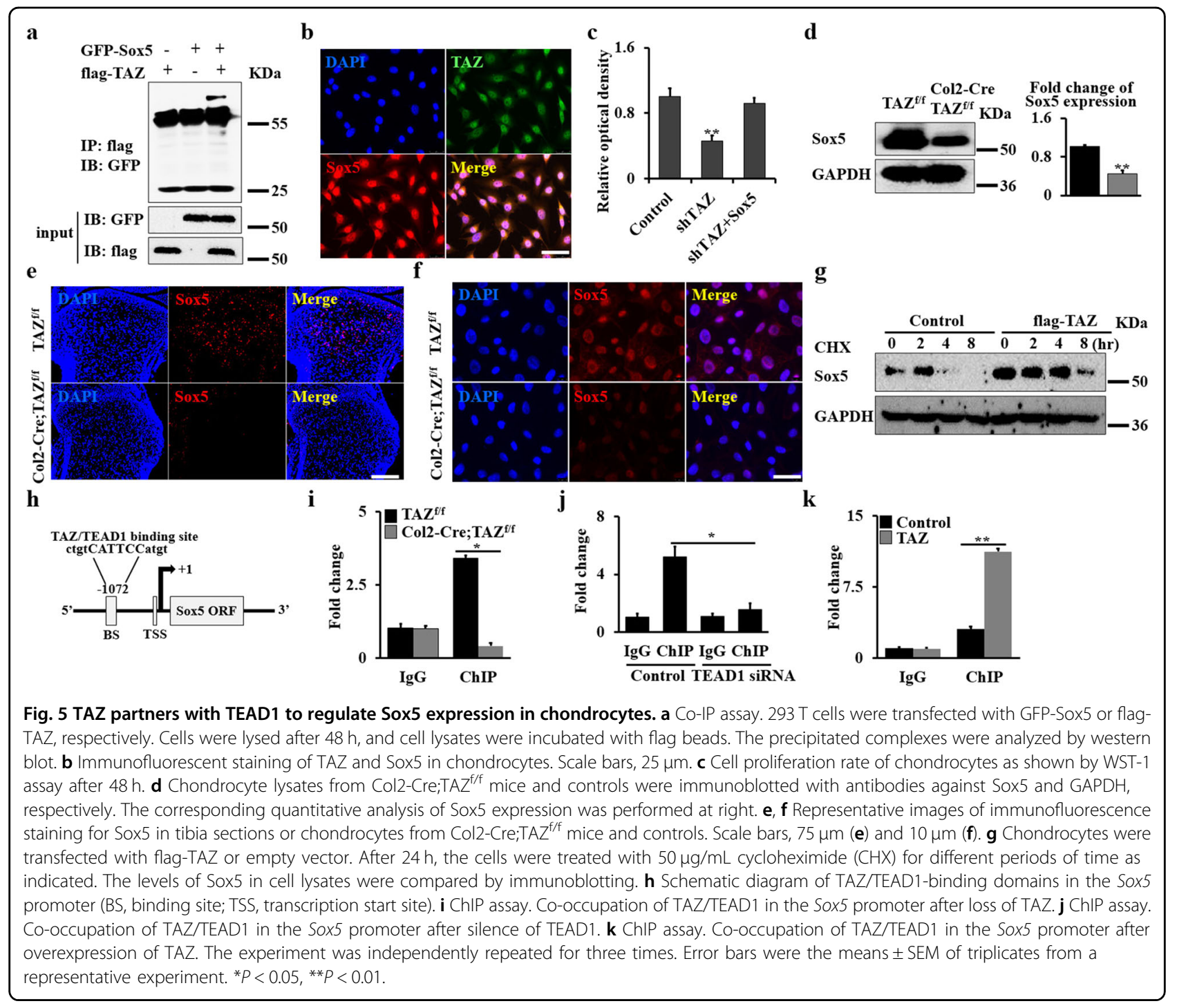

of bone from the callus tissues significantly decreased due to loss of TAZ (Fig. 7c). In addition, Alcian blue staining results showed that the cartilage area was much smaller in Col2-Cre; TAZ ${ }^{\mathrm{f} / \mathrm{f}}$ mice in comparison to the controls (Fig. $7 \mathrm{~d}, \mathrm{e}$ ). In addition, the expression levels of the chondrocyte-lineage genes Sox5, Col2a1, Col9a1, Col10a1, and aggrecan were all decreased at day 14 following the fracture in the Col2-Cre; $\mathrm{TAZ}^{\mathrm{f} / \mathrm{f}}$ mice compared to controls (Fig. $7 \mathrm{f}$ and Supplementary Fig. S6). Moreover, the immunofluorescence staining results showed a decreased Runx 2 and Col10a1 ${ }^{+}$signaling in the cartilage area of fracture callus compared to that in the control mice (Fig. 7g, h). Overall, these findings demonstrated that TAZ is a positive regulator of fracture healing.

\section{Discussion}

Although TAZ plays crucial roles in regulating the lineage commitment of MSCs to the differentiation of osteoblasts and adipocytes ${ }^{39,40}$, it is unknown about the effect of TAZ on chondrocytes and endochondral ossification. In this study, we demonstrated the essential role and mechanism of TAZ in cartilage and bone development, as well as fracture healing. We found that loss of TAZ inhibits the proliferation and differentiation of chondrocytes and the expression of cartilage matrix genes, which further impairs extracellular matrix proteins' production, eventually causing mouse growth retardation with impaired cartilage and bone formation. This may be caused by the failure in the generation of sufficient mature chondrocytes and production of adequate extracellular matrix needed to lead to defective cartilage development and function. TAZ ablation caused trabecular bone mass decrease may also result from defective chondrocyte differentiation and maturation, which blocks osteoblast differentiation and bone development due to inhibiting Runx2 signaling for promoting osteogenesis during 


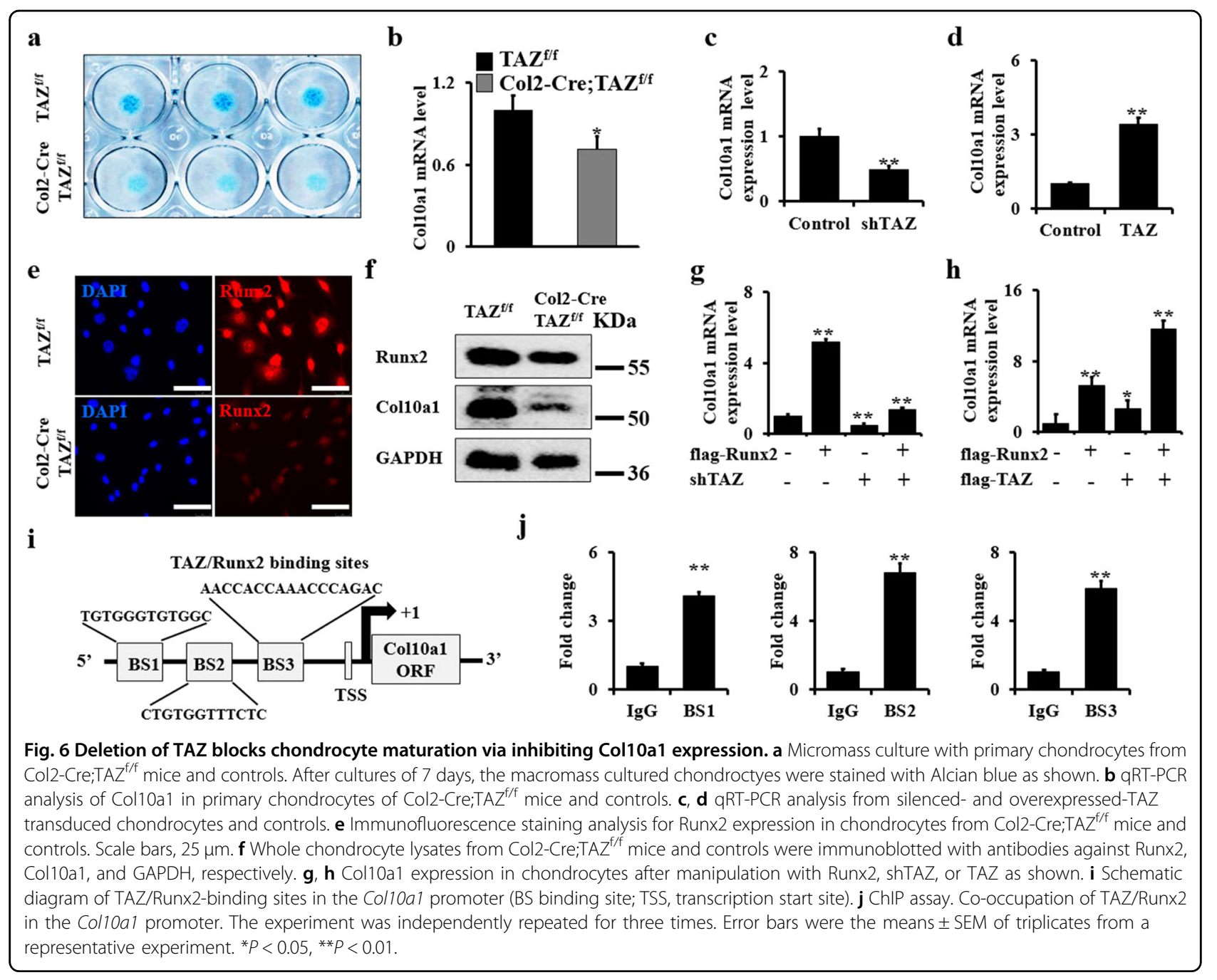

chondrocyte maturation stage. It is also possible that TAZ directly affects trabecular bone formation given that Col2Cre could also induce slightly TAZ deletion in osteoblasts. Therefore, the decreased trabecular bone mass could be from the impaired osteogenesis. These pathological changes are similar to those in Col2-Cre; $\mathrm{YAP}^{\mathrm{f} / \mathrm{f}}$ mice, which also exhibit the cartilage disruption and OA-like abnormality $^{21}$. Different from YAP ablation, we found that deletion of TAZ downregulates Col10a1 and MMP13. Our findings provide the first evidence that TAZ is required for cartilage and bone development, and provide new insight into development of promising therapeutic targets for skeleton abnormalities, heterotopic ossification, defective fracture healing, OA, and other bone diseases.

The Hippo-YAP/TAZ signaling pathway is an evolutionarily conserved pathway that controls the organ size and tumorigenesis by regulating cell proliferation, differentiation, apoptosis, and stem cell self-renewal ${ }^{39,41,42}$. Loss of the large tumor suppressor kinases $1 / 2$ (Lats1/2) or mammalian Ste20-like kinases $1 / 2$ (Mst1/2), the upstream components of Hippo pathway, impaired chondrocyte differentiation and caused chondrodysplasia, suggesting that the Hippo pathway plays an important role in the regulation of skeletal development ${ }^{12,43,44}$. TAZ is a major downstream effector of the Hippo pathway ${ }^{39}$. It has been reported to maintain the balance between osteogenesis and adipogenesis by regulating the expression of Runx2-mediated target genes to promote osteogenesis and suppressing the transcriptional activity of PPARy to inhibit adipogenesis ${ }^{22,24,25}$. In this study, we found that TAZ expression is gradually increased during chondrogenic differentiation. More importantly, we found that the loss of TAZ caused growth retardation, impaired endochondral ossification, and spontaneously developed OA-like phenotype. These findings are supported by the previous studies showing that deletion of YAP in chondrocytes, a paralog of TAZ, leads to a chondrodysplasia phenotype $^{12}$. However, recent studies reported that the roles of YAP and TAZ in bone are opposite ${ }^{24,45-47}$. In 


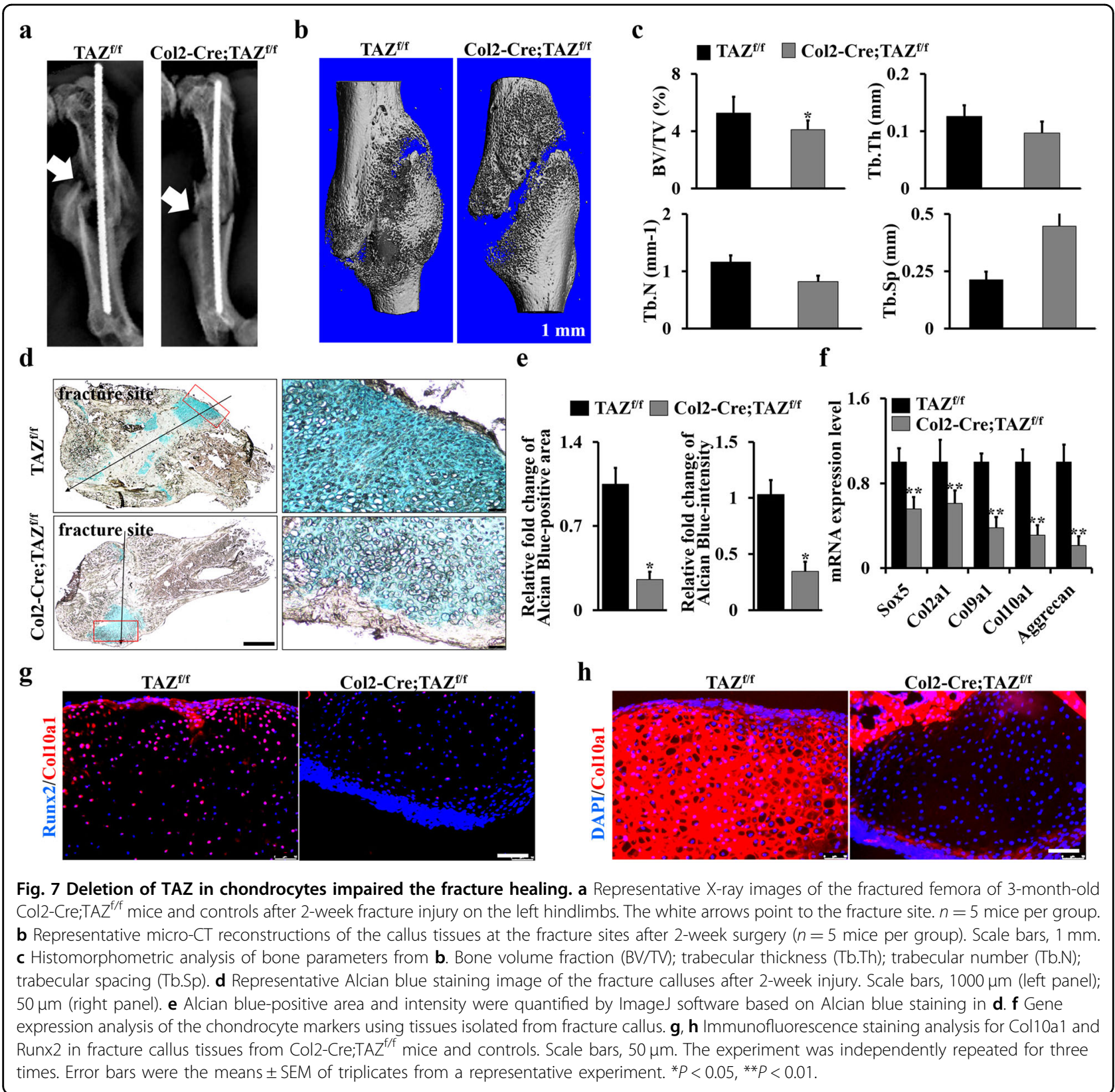

contrast, Christopher D. Kegelman et al. reported that YAP and TAZ have a similar function, combinatorically promoting the formation and development of bone by regulating osteoblast activity and osteoclastic remodeling $^{28}$. In consistent with that, our results showed that forced expression of TAZ promotes chondrocyte differentiation and upregulates the expression of chondrocyte markers, indicating that TAZ positively regulates chondrogenesis. Interestingly, YAP was reported to be decreased during chondrogenic differentiation and promote the chondrocyte proliferation ${ }^{12}$. Our heatmap data also found YAP expression shows a gradual decrease during chondrogenic differentiation. Overall, it is largely unknown how the Hippo pathway precisely coordinates the differential effects of YAP and TAZ in the regulation of osteoblast, chondrocyte, and adipocyte lineages, which needs to be further studied.

Our results showed the deletion of TAZ in chondrocyte lineage decreased expression of Col10a1 and Mmp13; however, the inhibitory effect of TAZ on Col10a1 expression was reduced at 4 months compared to that at 1 month. Consistent with our findings, Tokio Matsuzaki et al. reported that Col2-Cre;FoxO1/3/4 mice displayed articular defect phenotype, and the expression of Col10a1 


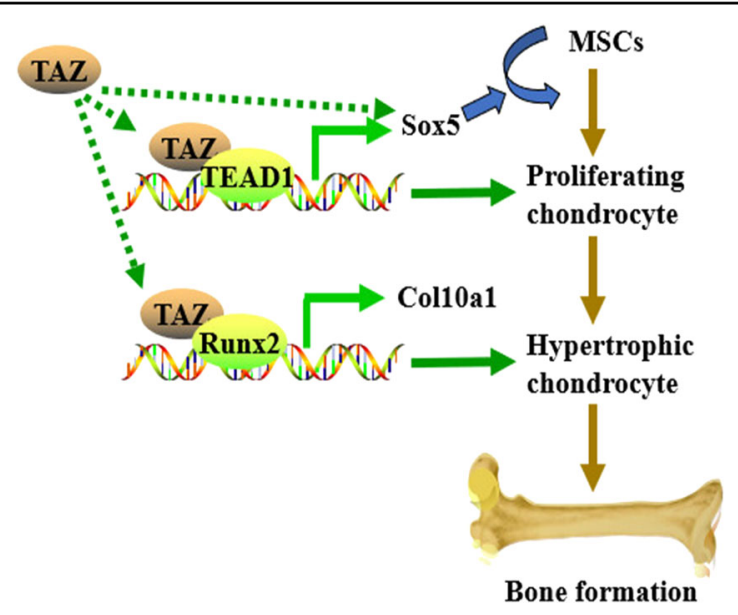

Fig. 8 A schematic presentation of the functions of TAZ on endochondral ossification and osteoarthritis development. TAZ promotes the proliferation and differentiation of chondrocytes by regulating the expression and stability of Sox5, and enhances chondrocyte maturation by regulating the Col10a1 expression.

and Mmp13 was decreased in the knee joint cartilage and thickness of articular cartilage was increased in 1-monthold Col2-Cre;FoxO1/3/4 mice compared to the controls ${ }^{48}$. Moreover, deletion of Mmp13 using Col2-Cre ${ }^{\text {ERT }}$ could also cause impaired articular cartilage and OA-like phenotype $^{49}$. Controversially, some studies have shown that OA causes increased expression of Col10a1 and Mmp13 in chondrocytes and disrupted articular cartilage $e^{21,50}$. Thus, these findings indicated that there may be a complicated relationship between Col10a1 and Mmp13 expression in cartilage and bone development and OA pathogenesis. Moreover, our results showed that upon loss of TAZ in chondrocytes at 1-month old, the articular cartilage is slightly thicker, which is supported by our further findings that deletion of TAZ inhibited chondrocyte proliferation; however, the cell apoptosis decreased during early stage of cartilage and bone development. Comprehensively understanding how TAZ affects cartilage matrix synthesis and function, as well as OA pathogenesis needs to be further investigated in the future.

Our result suggested that TAZ promotes chondrocyte proliferation and differentiation. These findings are consistent with the results reported by Deng et al. that YAP plays key roles in regulation of chondrocytes formation and cartilage maintenance ${ }^{12,21}$. Moreover, we found that TAZ drives chondrogenesis by regulating Sox 5 expression and stability, and chondrocyte maturation by induction of Col10a1 expression through association with Runx2. TAZ is one of key genes of the Hippo pathway and is a cofactor of the transcriptional factors, such as TEAD, Runx2, Smad, and Sox, and it cannot directly bind to its target genes' promoter to regulate their expression due to lack of
DNA-binding motif. We found that Sox 5 associated with TAZ and colocalized in nucleus of chondrocytes. Moreover, Sox 5 overexpression could reverse the cell growth in TAZ knockdown chondrocytes; while shSox 5 downregulates TAZ expression. These findings indicated that Sox 5 likely affects TAZ target genes through regulating TAZ expression. Our data also revealed that TAZ regulates Sox 5 expression, suggesting that there may be a loop between TAZ and Sox5. Although it has been reported that Col2-Cre transgenic mice have limited recombination capacity postnatally in cartilage tissues ${ }^{51}$, our result showed that in the fracture callus, TAZ level is effectively deleted in Col2-Cre;TAZ ${ }^{\mathrm{f} / \mathrm{f}}$ mice. In addition, we found that TAZ level has no pronounced change in the cortical bone of Col2-Cre; TAZ ${ }^{\mathrm{f} / \mathrm{f}}$ mice compared to controls. Thus, the defective facture healing likely results from the postnatal TAZ deletion. However, the results cannot exclude the secondary effect from the pronounced effects of TAZ deletion on cartilage and bone, which will be further confirmed by using the inducible Col2-Cre ${ }^{\text {ERT}}$; $\mathrm{TAZ}^{\mathrm{f} / \mathrm{f}}$ mouse model in the future study. Our data revealed that TAZ promotes fracture repair by regulating cartilaginous callus formation. Taken together, the findings from this study regarding the dual regulation nature of TAZ-derived chondrocyte differentiation and maturation in physiological and developmental contexts pave the way for new therapeutics against bone disease (Fig. 8).

In conclusion, this study provides new evidence that TAZ plays a critical role in cartilage and bone development and articular cartilage homeostasis through regulating chondrocyte proliferation, differentiation, and maturation. Loss of TAZ caused impaired cartilage formation and bone development. Thus, this study reveals that TAZ may be a potential drug target that can be utilized against bone and cartilage diseases.

\section{Materials and methods \\ Antibodies and reagents}

Antibodies from Cell Signaling Technology used in this study were against TAZ (\#83669; dilution 1:1000), Runx2 (\#12556; dilution 1:1000), and GAPDH (\#5174; dilution 1:1000). BrdU (\#sc-32323; dilution 1:1000), flag (\#sc-7945; dilution 1:1000), and GFP (\#sc-9996; dilution 1:1000) antibodies were obtained from Santa Cruz Biotechnology. Sox5 (\#PIPA5110416; dilution 1:1000) and Col10a1 (\#149771-80; dilution 1:1000) antibodies were purchased from Fisher Scientific ${ }^{\mathrm{TM}}$. The fluorescent secondary antibodies used were mouse and rabbit Alexa Fluor 594 and Alexa Fluor 647, all raised in goat and obtained from Abcam. TEAD1 siRNA was purchased from Santa Cruz Biotechnology. CHX was obtained from Sigma-Aldrich. BrdU labeling, calcein labeling, and EDTA-free cocktail inhibitor tablets, together with the other regents, were obtained from Fisher Scientific ${ }^{\mathrm{TM}}$. 


\section{Animals}

All protocols were approved by the Institutional Animal Care and Use Committees at the University of Pennsylvania and complied with the National Research Council's Guide for the Care and Use of Laboratory Animals. Col2Cre (Stock No: 003554) and $\mathrm{YAP}^{\mathrm{f} / \mathrm{f}} / \mathrm{TAZ}^{\mathrm{f} / \mathrm{f}}$ mice (Stock No: 030532) were ordered from The Jackson Laboratory (Bar Harbor, MA, USA), and the $\mathrm{TAZ}^{\mathrm{f} / \mathrm{f}}$ mice were obtained by separating the $\mathrm{YAP}^{\mathrm{f} / \mathrm{f}} / \mathrm{TAZ}^{\mathrm{f} / \mathrm{f}}$ mice.

\section{Cell culture}

Primary chondrocytes were isolated from the limb buds of embryo at E15.5. Briefly, the embryonic limb buds from WT, $\mathrm{TAZ}^{\mathrm{f} / \mathrm{f}}$, or Col2-Cre;TAZ ${ }^{\mathrm{f} / \mathrm{f}}$ mice at E15.5 was dissociated with Trypsin solution (Fisher Scientific ${ }^{\mathrm{TM}}$, USA) by incubation for $30 \mathrm{~min}$ at $37^{\circ} \mathrm{C}$, and then washed the cells for two times with PBS and suspended in $\alpha$-Minimum Essential Medium ( $\alpha$-MEM; Gibco, USA $)^{52}$. Primary chondrocytes were cultured in $\alpha$-MEM supplemented with Pen-Strep and 10\% FBS (Gibco, USA). The $293 \mathrm{~T}$ cell line was cultured in DMEM (Gibco, USA) supplemented with Pen-Strep and 10\% FBS. All of these cells were incubated at $37^{\circ} \mathrm{C}$ with $5 \%$ humidified $\mathrm{CO}_{2}$, and the medium was added and replaced every 3 days. Micromass cultures were performed as previously described ${ }^{53}$.

\section{Plasmids, transfection, and lentiviral infection}

The plasmids flag-TAZ (\#27318), GFP-Sox5 (\#48707), and shTAZ (\#31795) were obtained from Addgene; and shSox 5 (i042548a) was from ABM. Plasmid transfection was carried out using FuGENE ${ }^{\circledR}$ HD Transfection Reagent (Promega, USA) according to the manufacturer's instructions. Lentiviral infection was carried out as descried previously $^{54}$. Briefly, $293 \mathrm{~T}$ cells were first co-transfected with shSox 5 and packaging plasmids. The progeny lentiviral released from the co-transfected $293 \mathrm{~T}$ cells were filtered, collected, and used to infect the chondrocytes.

\section{qRT-PCR}

Total RNA was extracted using TRIzol reagent (Invitrogen, USA), and $2 \mu \mathrm{g}$ of the total RNA was retrotranscribed into cDNA using the PrimeScript ${ }^{\mathrm{TM}} \mathrm{RT}$ Reagent Kit (TaKaRa, Japan). Subsequently, qRT-PCR was carried out in a CFX96 Real-Time PCR System with the SYBR Green Master PCR mix (Bimake, USA). GAPDH served as an internal control. The primers used for the quantification are listed in Supplementary Table S1.

\section{Cellular functional assay}

For the proliferative test, the WST-1 assay was performed using the WST-1 Cell Proliferation Assay Kit (Cayman Chemical, USA) according to the manufacturer's instructions. Briefly, logarithmically growing cells were trypsinized, and $5 \times 10^{3}$ cells in $1 \mathrm{~mL}$ of cell culture medium were seeded in triplicate in 96-well plates, and then the $\mathrm{OD}_{490}$ was measured after 24, 48, and $72 \mathrm{~h}$ in culture.

For the colony formation assay, we seeded $5 \times 10^{3}$ chondrocytes in six-well plates and cultured the cells for 5 days. Subsequently, the crystal violet staining was carried out to count the colony number, and the triplicate plates were counted.

\section{Radiographic procedures and bone micro-CT analysis}

Radiographic procedures were performed in the Siemens X-ray equipment (Madison, WI, USA). The bone morphology and microarchitecture were carried out and analyzed using a micro-CT system (Images acquired at $55 \mathrm{kVp}$ energy, $145 \mathrm{~mA}$, and $300 \mathrm{~ms}$ integration time; School of Medicine, University of Pennsylvania, USA) ${ }^{54}$.

\section{Histology}

Femurs and tibiae were harvested, fixed in 4\% PFA overnight, decalcified for 1 month in 10\% EDTA, and then paraffin-embedded. Six-micrometer sections were cut, and Hemotoxylin and Eosin, Safranin O/fast green, and Alcian blue staining were performed, as we previously described $^{54-56}$.

\section{Skeletal staining and analysis}

Whole mouse carcasses were collected from newborns after euthanasia, fixed for 1 day in $100 \%$ ethanol, defatted for 1 day in acetone at room temperature (RT), stained sequentially with Alcian blue for 1 day and Alizarin red $\mathrm{S}$ in $1 \% \mathrm{KOH}$ for $2 \mathrm{~h}$, washed with the mixing solution of $1 \%$ $\mathrm{KOH}$ and $20 \%$ glycerol for several times, and finally stored in 50\% ethanol and 50\% glycerol.

\section{Calcein labeling}

Calcein $(20 \mathrm{mg} / \mathrm{kg})$ labeling was performed 1 and 5 days before 2-month-old Col2-Cre;TAZ ${ }^{\mathrm{f} / \mathrm{f}}$, and control mice were sacrificed. At the end of the experiments, tibiae were collected, fixed in $4 \%$ PFA, and embedded. The sections were cut at $6-\mu \mathrm{m}$ thickness and viewed under a fluorescence microscope. The BFR per bone surface $\left(\mu \mathrm{m}^{3} / \mu \mathrm{m}^{2}\right.$ per day) and MAR ( $\mu \mathrm{m}$ per day) were analyzed by the OsteoMeasure analysis system, as we previously reported ${ }^{56,57}$.

\section{Immunofluorescence and immunohistochemistry staining}

For chondrocyte immunofluorescence staining, chondrocytes cultured on coverslips were fixed with 4\% PFA and permeabilized with TBST (1\%o Triton X-100 in TBS). Nonspecific binding of antibodies to cells was blocked by incubation at RT for $1 \mathrm{~h}$ in TBST including 3\% BSA, and then the corresponding primary antibodies (1:500 dilution) were added to incubate with cells overnight at $4{ }^{\circ} \mathrm{C}$. Subsequently, chondrocytes were washed three times with TBST and incubated with Alexa Fluor 594- or Alexa Fluor 647conjugated secondary antibodies for $1 \mathrm{~h}$ at RT. Nuclei were 
counterstained with 4,6-diamidino-2-phenylindole (DAPI) and washed three times with TBST. Then, the cells were mounted and visualized using a fluorescence microscope.

For tissue immunohistochemistry staining, tissues previously fixed in $4 \%$ PFA were dehydrated through serial incubations in $75 \%, 95 \%$, and $100 \%$ ethanol and $100 \%$ xylene, and then embedded in the melted paraffin. Sixmicron sections were mounted onto slides and deparaffinized. Endogenous peroxidase was inactivated by $3 \%$ $\mathrm{H}_{2} \mathrm{O}_{2}$ at $\mathrm{RT}$ for $10 \mathrm{~min}$. The sections were blocked at RT for $1 \mathrm{~h}$ in goat serum. Subsequently, the primary antibody (1:500 dilution) was applied at RT for $1 \mathrm{~h}$. After washing three times with TBST, the sections were incubated with the corresponding secondary antibody (1:1000 dilution) at RT for $1 \mathrm{~h}$. After washing with TBST, the sections were mounted and visualized using a fluorescence microscope.

\section{In situ detection of apoptotic cells}

TUNEL assay was performed as described ${ }^{56}$. Briefly, the sections were permeabilized with $0.1 \%$ Triton X-100, then incubated with TUNEL reaction mixture (label solution and enzyme solution) for $1 \mathrm{~h}$ at $37^{\circ} \mathrm{C}$ avoiding light. After rinsing the sections three times in PBS for $5 \mathrm{~min}$, the nuclei were counterstained with DAPI and mounted, and visualized using a fluorescence microscope.

\section{ChIP assay}

ChIP assays were carried out using the Imprint Chromatin Immunoprecipitation Kit (Sigma, USA) according to the manufacturer's instructions ${ }^{58}$.

\section{Western blot and Co-IP}

Cells were lysed in modified RIPA lysis buffer supplemented with a protease cocktail inhibitor (Fisher Scientific $^{\mathrm{TM}}$, USA). The concentration of protein was determined using the Pierce BCA Protein Assay Kit (Thermo Fisher, USA). Twenty micrograms of protein from each sample was subjected to sodium dodecyl sulfate-polyacrylamide gel electrophoresis (SDS-PAGE), transferred to a polyvinylidene fluoride membrane (Millipore, USA), and immunoblotted with various antibodies. Following overnight incubation at $4{ }^{\circ} \mathrm{C}$, the membranes were washed and then incubated with the corresponding HRP-conjugated secondary antibodies for $1 \mathrm{~h}$ at RT. The membranes were then detected by chemiluminescence with ECL (Thermo Fisher, USA).

Regarding Co-IP, the $293 \mathrm{~T}$ cells were transfected with various expression vectors by using the FuGENE ${ }^{\circledR} \mathrm{HD}$ transfection reagent (Promega, USA), according to the manufacturer's instructions with slight modification. Briefly, the $293 \mathrm{~T}$ cells were first lysed with IP buffer, and its supernatants were collected and incubated with antiGFP antibody, anti-flag antibody, and protein A/G agarose (Sigma, USA). After washing three times with TBST at $4{ }^{\circ} \mathrm{C}$, the immune complexes were subjected to SDS-PAGE, and then analyzed by western blot.

\section{Bioinformatic analysis}

Publicly available microarray datasets from Leila Taher et al. $^{32}$ (GSE30318) was used to determine whether select TAZ-associated genes (LATS1, MST1, MOB1, YAP, CYR61, and CTGF) were differentially expressed at five stages of limb development (from E9.5 to 13.5). All data were analyzed using R, version 3.6.2. Histological scoring of OA is performed using the Osteoarthritis Research Society International (OARSI) scoring system ${ }^{59}$. Synovial scores are evaluated as described ${ }^{48,60}$.

\section{Statistical analysis}

All statistical analyses were carried out using the SPSS21 statistical software package. Data were analyzed by Student's $t$-test to determine statistically significant differences between groups. $P$ values $<0.05$ were considered significant.

\section{Acknowledgements}

We are thankful to the animal facility at University of Pennsylvania. This study was supported by the National Institute of Arthritis and Musculoskeletal and Skin Diseases (NIAMS, AR061052), the National Institute of Aging (NIA, AG048388), and the National Institute of Dental and Craniofacial Research (NIDCR, DE023105) awarded to Shuying Yang.

\section{Author details \\ ${ }^{1}$ Department of Basic \& Translational Sciences, School of Dental Medicine, University of Pennsylvania, Philadelphia, PA 19104, USA. ${ }^{2}$ Department of Orthopedic Surgery, Perelman School of Medicine, University of Pennsylvania, Philadelphia, PA 19104, USA. ${ }^{3}$ The Penn Center for Musculoskeletal Disorders, School of Medicine, University of Pennsylvania, Philadelphia, PA 19104, USA. \\ ${ }^{4}$ Center for Innovation \& Precision Dentistry, School of Dental Medicine, School of Engineering and Applied Sciences, University of Pennsylvania, Philadelphia, PA 19104, USA}

\section{Author contributions}

Shuying Yang and Y.L. conceived this study, generated hypotheses, and designed experiments. Y.L., Shuting Yang, and L.Q. performed experiments and analyzed data. Shuying Yang and Y.L. wrote, reviewed, and edited the paper. Shuying Yang supervised the project.

\section{Conflict of interest}

The authors declare no competing interests.

\section{Publisher's note}

Springer Nature remains neutral with regard to jurisdictional claims in published maps and institutional affiliations.

Supplementary information The online version contains supplementary material available at https://doi.org/10.1038/s41421-021-00254-5.

Received: 24 July 2020 Accepted: 16 February 2021

Published online: 20 April 2021

\section{References \\ 1. Zuscik, M. J., Hilton, M. J., Zhang, X., Chen, D. \& O'Keefe, R. J. Regulation of chondrogenesis and chondrocyte differentiation by stress. J. Clin. Investig. 118,} 429-438 (2008). 
2. Aghajanian, P. \& Mohan, S. The art of building bone: emerging role of chondrocyte-to-osteoblast transdifferentiation in endochondral ossification. Bone Res. 6, 19 (2018).

3. Goldring, M. B. Chondrogenesis, chondrocyte differentiation, and articular cartilage metabolism in health and osteoarthritis. Ther. Adv. Musculoskelet. Dis. 4, 269-285 (2012)

4. Jing, Y. et al. Chondrogenesis and osteogenesis are one continuous developmental and lineage defined biological process. Sci. Rep. 7, 10020 (2017)

5. Ono, N., Ono, W., Nagasawa, T. \& Kronenberg, H. M. A subset of chondrogenic cells provides early mesenchymal progenitors in growing bones. Nat. Cell Biol. 16, 1157-1167 (2014).

6. Kobayashi, T. et al. Dicer-dependent pathways regulate chondrocyte proliferation and differentiation. Proc. Natl Acad. Sci. USA 105, 1949-1954 (2008).

7. Lian, W. S. et al. MicroRNA-128a represses chondrocyte autophagy and exacerbates knee osteoarthritis by disrupting Atg12. Cell Death Dis. 9, 919 (2018),

8. Heidari, B. Knee osteoarthritis prevalence, risk factors, pathogenesis and features: part I. Casp. J. Intern. Med. 2, 205-212 (2011)

9. Loeser, R. F., Goldring, S. R., Scanzello, C. R. \& Goldring, M. B. Osteoarthritis: a disease of the joint as an organ. Arthritis Rheum. 64, 1697-1707 (2012).

10. Toh, W. S. et al. Cartilage repair using hyaluronan hydrogel-encapsulated human embryonic stem cell-derived chondrogenic cells. Biomaterials $\mathbf{3 1}$ 6968-6980 (2010)

11. Baboolal, T. G. et al. Synovial fluid hyaluronan mediates MSC attachment to cartilage, a potential novel mechanism contributing to cartilage repair in osteoarthritis using knee joint distraction. Ann. Rheum. Dis. 75, 908-915 (2016).

12. Deng, $Y$, et al. Yap1 regulates multiple steps of chondrocyte differentiation during skeletal development and bone repair. Cell Rep. 14, 2224-2237 (2016).

13. Liu, C. F., Samsa, W. E., Zhou, G. \& Lefebvre, V. Transcriptional control of chondrocyte specification and differentiation. Semin. Cell Dev. Biol. 62, 34-49 (2017).

14. Li, J. \& Dong, S. The Signaling pathways involved in chondrocyte differentiation and hypertrophic differentiation. Stem Cells Int. 2016, 2470351 (2016).

15. Wu, C. et al. Kindlin-2 controls TGF-beta signalling and Sox9 expression to regulate chondrogenesis. Nat. Commun. 6, 7531 (2015)

16. Goldring, M. B., Tsuchimochi, K. \& ljiri, K. The control of chondrogenesis. J. Cell Biochem. 97, 33-44 (2006).

17. Meng, Z., Moroishi, T. \& Guan, K. L. Mechanisms of Hippo pathway regulation Genes Dev. 30, 1-17 (2016)

18. Yu, F. X. \& Guan, K. L. The Hippo pathway: regulators and regulations. Genes Dev. 27, 355-371 (2013).

19. Harvey, K. F., Zhang, X. \& Thomas, D. M. The Hippo pathway and human cancer. Nat. Rev. Cancer 13, 246-257 (2013).

20. Yang, $W$. et al. The emerging role of Hippo signaling pathway in regulating osteoclast formation. J. Cell Physiol. 233, 4606-4617 (2018).

21. Deng, Y. et al. Reciprocal inhibition of YAP/TAZ and NF-kappaB regulates osteoarthritic cartilage degradation. Nat. Commun. 9, 4564 (2018).

22. Xiao, Z. et al. Polycystin-1 interacts with TAZ to stimulate osteoblastogenesis and inhibit adipogenesis. J. Clin. Invest. 128, 157-174 (2018).

23. Matsumoto, $Y$. et al. Reciprocal stabilization of $A B L$ and $T A Z$ regulates osteoblastogenesis through transcription factor RUNX2. J. Clin. Invest. 126 4482-4496 (2016).

24. Hong, J. H. et al. TAZ, a transcriptional modulator of mesenchymal stem cell differentiation. Science 309, 1074-1078 (2005).

25. Byun, M. R. et al. TAZ is required for the osteogenic and anti-adipogenic activities of kaempferol. Bone 50, 364-372 (2012).

26. Yang, J. Y. et al. Osteoblast-targeted overexpression of TAZ increases bone mass in vivo. PLOS ONE 8, e56585 (2013).

27. Pappalardo, A. et al. Thyroid development in zebrafish lacking Taz. Mech. Dev. 138, Pt 3, 268-278 (2015).

28. Kegelman, C. D. et al. Skeletal cell YAP and TAZ combinatorially promote bone development. FASEB J. 32, 2706-2721 (2018)

29. Makita, R. et al. Multiple renal cysts, urinary concentration defects, and pulmonary emphysematous changes in mice lacking TAZ. Am. J. Physiol. Renal. Physiol. 294, F542-F553 (2008).

30. Yang, B. et al. YAP1 negatively regulates chondrocyte differentiation partly by activating the beta-catenin signaling pathway. Int. J. Biochem. Cell Biol. 87 104-113 (2017).

31. Karystinou, $A$. et al. Yes-associated protein (YAP) is a negative regulator of chondrogenesis in mesenchymal stem cells. Arthritis Res. Ther. 17, 147 (2015).

32. Taher, L. et al. Global gene expression analysis of murine limb development. PLOS ONE 6, e28358 (2011)
33. Varelas, X. et al. TAZ controls Smad nucleocytoplasmic shuttling and regulates human embryonic stem-cell self-renewal. Nat. Cell Biol. 10, 837-848 (2008).

34. Tang, Y., Feinberg, T., Keller, E. T., Li, X. Y. \& Weiss, S. J. Snail/Slug binding interactions with YAP/TAZ control skeletal stem cell self-renewal and differentiation. Nat. Cell Biol. 18, 917-929 (2016).

35. Zou, $\mathrm{H}$. et al. SOX5 interacts with YAP1 to drive malignant potential of nonsmall cell lung cancer cells. Am. J. Cancer Res. 8, 866-878 (2018).

36. Lefebvre, V., Behringer, R. R. \& de Crombrugghe, B. L-Sox5, Sox6 and Sox9 control essential steps of the chondrocyte differentiation pathway. Osteoarthr. Cartil. 9(Suppl A), S69-S75 (2001).

37. Ikeda, T. et al. Distinct roles of Sox5, Sox6, and Sox9 in different stages of chondrogenic differentiation. J. Bone Miner. Metab. 23, 337-340 (2005).

38. Li, F. F. et al. Runx2 contributes to murine Col10a1 gene regulation through direct interaction with its cis-enhancer. J. Bone Miner. Res. 26, 2899-2910 (2011).

39. Mo, J. S., Park, H. W. \& Guan, K. L. The Hippo signaling pathway in stem cell biology and cancer. EMBO Rep. 15, 642-656 (2014).

40. Panciera, T. et al. Induction of expandable tissue-specific stem/progenitor cells through transient expression of YAP/TAZ. Cell Stem Cell 19, 725-737 (2016).

41. Pan, D. Hippo signaling in organ size control. Genes Dev. 21, 886-897 (2007).

42. Mohri, Z., Del Rio Hernandez, A. \& Krams, R. The emerging role of YAP/TAZ in mechanotransduction. J. Thorac. Dis. 9, E507-E509 (2017).

43. Song, $\mathrm{H}$. et al. Ablation of Rassf2 induces bone defects and subsequent haematopoietic anomalies in mice. EMBO J. 31, 1147-1159 (2012).

44. Ni, L. S., Zheng, Y. G., Hara, M., Pan, D. J. \& Luo, X. L. Structural basis for Mob1dependent activation of the core Mst-Lats kinase cascade in Hippo signaling. Genes Dev. 29, 1416-1431 (2015).

45. Xiong, J. H., Almeida, M. \& O'Brien, C. A. The YAP/TAZ transcriptional coactivators have opposing effects at different stages of osteoblast differentiation. Bone 112, 1-9 (2018)

46. Zaidi, S. K. et al. Tyrosine phosphorylation controls Runx2-mediated subnuclear targeting of YAP to repress transcription. EMBO J. 23, 790-799 (2004).

47. Hong, J. H. \& Yaffe, M. B. TAZ - A beta-catenin-like molecule that regulates mesenchymal stem cell differentiation. Cell Cycle 5, 176-179 (2006).

48. Matsuzaki, T. et al. FoxO transcription factors modulate autophagy and proteoglycan 4 in cartilage homeostasis and osteoarthritis. Sci. Transl. Med. 10, eaan0746 (2018).

49. Wang, M. N. et al. MMP13 is a critical target gene during the progression of osteoarthritis. Arthritis Res. Ther. 15, R5 (2013).

50. Corciulo, C. et al. Endogenous adenosine maintains cartilage homeostasis and exogenous adenosine inhibits osteoarthritis progression. Nat. Commun. 8, 15019 (2017)

51. Stiers, P. J., van Gastel, N., Moermans, K., Stockmans, I. \& Carmeliet, G. Regulatory elements driving the expression of skeletal lineage reporters differ during bone development and adulthood. Bone 105, 154-162 (2017).

52. Chen, Y., Xu, H. \& Lin, G. Generation of iPSC-derived limb progenitor-like cells for stimulating phalange regeneration in the adult mouse. Cell Discov. 3, 17046 (2017).

53. Saha, A., Rolfe, R., Carroll, S., Kelly, D. J. \& Murphy, P. Chondrogenesis of embryonic limb bud cells in micromass culture progresses rapidly to hypertrophy and is modulated by hydrostatic pressure. Cell Tissue Res. 368, 47-59 (2017).

54. Yuan, X. et al. Ciliary IFT80 balances canonical versus non-canonical hedgehog signalling for osteoblast differentiation. Nat. Commun. 7, 11024 (2016).

55. Liu, M., Alharbi, M., Graves, D. \& Yang, S. IFT80 is required for fracture healing through controlling the regulation of tgf-beta signaling in chondrocyte differentiation and function. J. Bone Miner. Res. 35, 571-582 (2019).

56. Li, Z. et al. Regulator of $\mathrm{G}$ protein signaling protein 12 (Rgs12) controls mouse osteoblast differentiation via calcium channel/oscillation and galphai-ERK signaling. J. Bone Miner. Res. 34, 752-764 (2019).

57. $\mathrm{Ng}, \mathrm{A} . \mathrm{Y} . \mathrm{H}$. et al. Regulator of $\mathrm{G}$ protein signaling 12 enhances osteoclastogenesis by suppressing Nrf2-dependent antioxidant proteins to promote the generation of reactive oxygen species. Elife 8, e42951 (2019).

58. Li, Y., Hu, N., Yang, D., Oxenkrug, G. \& Yang, Q. Regulating the balance between the kynurenine and serotonin pathways of tryptophan metabolism. FEBS J. 284, 948-966 (2017).

59. Pritzker, K. P. et al. Osteoarthritis cartilage histopathology: grading and staging. Osteoarthritis. Cartilage. 14, 13-29 (2006)

60. Schelbergen, R. F. et al. Alarmins S100A8/S100A9 aggravate osteophyte formation in experimental osteoarthritis and predict osteophyte progression in early human symptomatic osteoarthritis. Ann. Rheum. Dis. 75, 218-225 (2016). 